\title{
The zero-energy state in graphene in a high magnetic field
}

\author{
Joseph G. Checkelsky, Lu Li and N. P. Ong \\ Department of Physics, Princeton University, Princeton, NJ 08544, USA
}

(Dated: October 27, 2018)

\begin{abstract}
The fate of the charge-neutral Dirac point in graphene in a high magnetic field $H$ has been investigated at low temperatures $(T \sim 0.3 \mathrm{~K})$. In samples with small gate-voltage offset $V_{0}$, the resistance $R_{0}$ at the Dirac point diverges steeply with $H$, signalling a crossover to an insulating state in high field. The approach to the insulating state is highly unusual. Despite the steep divergence in $R_{0}$, the profile of $R_{0}$ vs. $T$ in fixed $H$ saturates to a $T$-independent value below $2 \mathrm{~K}$, consistent with gapless charge-carrying excitations.
\end{abstract}

PACS numbers: 73.63.-b,73.21.-b,73.43.-f

The discovery of the quantum Hall effect (QHE) in monolayer graphene crystals provides a new system for investigating relativistic Dirac-like excitations in solids $[1,2,3,[4,[5,6]$. In a magnetic field $H$, the system forms Landau Levels (indexed by $n$ ) that are 4 -fold degenerate. The Hall conductivity $\sigma_{x y}$ is accurately quantized as the chemical potential $\mu$ is changed from the hole part to electron part of the Dirac spectrum. Considerable attention has focussed on the $n=0$ Landau Level (LL), especially on the nature of the electronic state at the charge-neutral point $(\mu=0)$ in an intense magnetic field $H$. Several groups [7, 8, 9, 10, 11, 12] have predicted that a large field stabilizes the quantum Hall ferromagnetic (QHF) state, in which the pseudospins describing the valley degree of freedom become ferromagnetically ordered. In a second group of theories [13, 14], interaction causes an excitonic gap to open at the Dirac point. Experiments are actively addressing these issues [15, 16, 17]. Jiang et al. [16] have inferred that the sublevel gaps at $\nu=0$ and \pm 1 arise from lifting of the spin and sub-lattice degeneracies, respectively, and inferred a many-body origin for the states. We have found that, in samples with small $V_{0}$ (the gate voltage needed to align $\mu$ with the Dirac point), the value $R_{0}$ of the resistance $R_{x x}$ at the Dirac point diverges steeply with $H$, i.e. a large $H$ drives the Dirac point insulating. Despite the strong $H$ dependence, $R_{0}$ saturates to a $T$-independent value below $2 \mathrm{~K}$, providing evidence for charged, gapless excitations. In samples with large $V_{0}$, this divergence in $R_{0}$ is shifted to higher fields.

Following Refs. 1, 2, 4], we peeled single-layer graphene crystals (3-10 $\mu \mathrm{m}$ in length) from Kish graphite on a $\mathrm{Si}_{-} \mathrm{SiO}_{2}$ wafer. $\mathrm{Au} / \mathrm{Cr}$ contacts were deposited using e-beam lithography (Fig. 1a, inset). We have found that the high-field behavior of $R_{0}$ is strongly correlated with $V_{0}$ (Table I). All samples (except K22) have $\mu$ lying in the electron band (positive $V_{0}$ ). Samples in which $\left|V_{0}\right|<1 \mathrm{~V}$ (K7 and K22) display a very large $R_{0}(14)$ (resistance measured at $14 \mathrm{~T}$ and $0.3 \mathrm{~K}$ ), which arises from the strong divergence mentioned. By contrast, in samples with large $\left|V_{0}\right|, R_{0}(14) \leq 7 \mathrm{k} \Omega$.

Figure 17 shows the variation of $R_{x x}$ in K7 plotted vs. the shifted gate voltage $V_{g}^{\prime}=V_{g}-V_{0}$ with $H$ held at 8,11 and $14 \mathrm{~T}$ (at $T=0.3 \mathrm{~K}$ ). The striking feature here is that the peak corresponding to the $n=0 \mathrm{LL}$ increases to $>100$ $\mathrm{k} \Omega$ at $14 \mathrm{~T}$, whereas the peaks corresponding to $n= \pm 1$ remain below $\sim 7 \mathrm{k} \Omega$. As in Refs. 11, 2, 3, 4, 5], the Hall conductivity $\sigma_{x y}$ (Panel b) displays plateaus given by [12]

$$
\sigma_{x y}=\frac{\nu e^{2}}{h}=\frac{4 e^{2}}{h}\left(n+\frac{1}{2}\right),
$$

where $n$ indexes the 4 -fold degenerate LL and $\nu$ indexes individual sublevels. In K7, the 'zero' plateau $\sigma_{x y} \simeq 0$ at $V_{g}^{\prime}=0$ is already visible at $H=8 \mathrm{~T}$.

Narrowing our focus to the $n=0 \mathrm{LL}$, we examine $R_{x x}$ in the $n=0$ LL as a function of $T$, with $H$ fixed at $14 \mathrm{~T}$ (Fig. 22 ). We see that, from 40 to $0.3 \mathrm{~K}, R_{0}$ rises steeply from $4 \mathrm{k} \Omega$ to $190 \mathrm{k} \Omega$. The curve of the conductivity $\sigma_{x x}$ plotted vs. $V_{g}^{\prime}$ reveals a 2 -peak structure that implies splitting of the 4-fold degeneracy by a gap $\Delta$ (Fig. 2b). At $100 \mathrm{~K}$, the two peaks are already resolved. With decreasing $T$, the minimum at $V_{g}^{\prime}=0$ initially deepens rapidly, but saturates below $\sim 2 \mathrm{~K}$. The Hall conductivity $\sigma_{x y}$ at $0.3 \mathrm{~K}$ (thin curve) displays a well-defined plateau on which $\sigma_{x y} \simeq 0$. Because the next plateau is at $\sigma_{x y}=2\left(e^{2} / h\right)$, we infer that each of the peaks in $\sigma_{x x}$ is comprised of 2 unresolved sublevels. The opening of the gap causes $\sigma_{x x}$ (at $V_{g}^{\prime}=0$ ) to fall rapidly with decreasing $T$, until saturation occurs below $2 \mathrm{~K}$.

The behavior of $R_{0}$ described here is qualitatively different from that in, for e.g., Ref. 15]. To understand the difference, we have examined several samples (Table \). As mentioned, the offset gate $V_{0}$ is a crucial parameter. Figure 2r compares the curves of $R_{x x}(n=0 \mathrm{LL})$ in the

\begin{tabular}{|c|c|c|c|}
\hline Sample & $V_{0}(\mathrm{~V})$ & $R_{0}(14)(\mathrm{k} \Omega)$ & $\mu_{e}(1 / \mathrm{T})$ \\
\hline $\mathrm{K} 5$ & 3 & 80 & 0.3 \\
$\mathrm{~K} 7$ & 1 & 190 & 1.3 \\
$\mathrm{~K} 8$ & 12 & 15 & 0.6 \\
$\mathrm{~K} 18$ & 20 & 7.5 & 0.9 \\
$\mathrm{~K} 22$ & -0.6 & $>280$ & 2.5 \\
$\mathrm{~K} 29$ & 22.5 & 7 & 0.2 \\
\hline
\end{tabular}

TABLE I: Sample parameters. $V_{0}$ is the gate voltage needed to bring $\mu$ to $0 . R_{0}(14)$ is $R_{0}$ measured at $H=14 \mathrm{~T}$ and $T$ $=0.3 \mathrm{~K} . \mu_{e}$ is the electron mobility at $H=0$. 


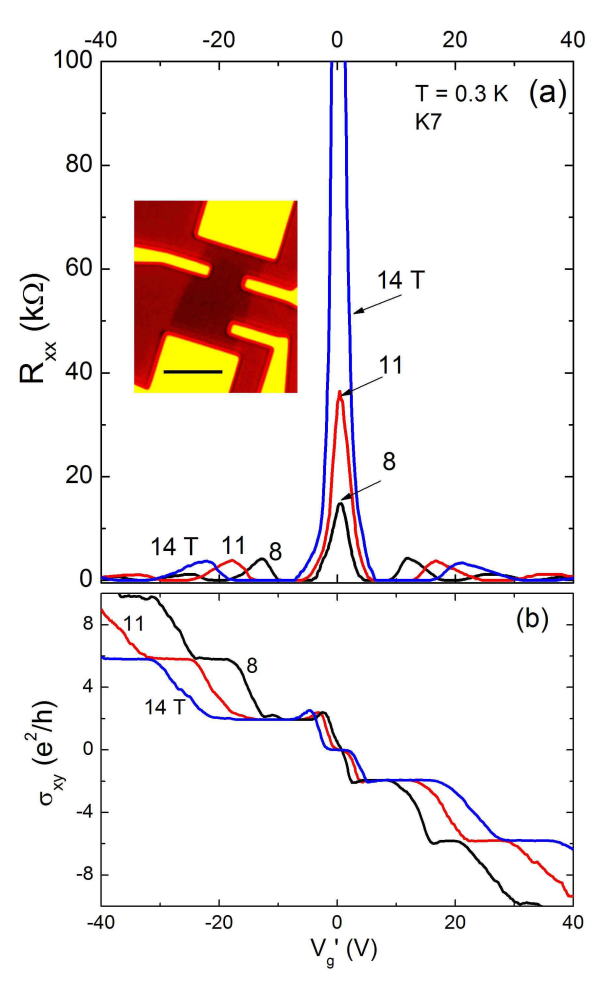

FIG. 1: (color online) The resistance $R_{x x}$ (Panel a) and Hall conductivity $\sigma_{x y}$ (b) in Sample K7 versus (shifted) gate voltage $V_{g}^{\prime}=V_{g}-V_{0}$ at $0.3 \mathrm{~K}$ with $H$ fixed at 8,11 and $14 \mathrm{~T}$. Peaks of $R_{x x}$ at finite $V_{g}^{\prime}$ correspond to the filling of the $n=1$ and $n=2$ LLs. At $V_{g}^{\prime}=0$, the peak in $R_{x x}$ grows to 190 $\mathrm{k} \Omega$ at $14 \mathrm{~T}$. The inset shows in false color a graphene crystal (dark red) with Au leads deposited (yellow regions). The bar indicates $5 \mu \mathrm{m}$. Panel b shows the quantization of $\sigma_{x y}$ at the values $\left(4 e^{2} / h\right)\left(n+\frac{1}{2}\right)$. At $0.3 \mathrm{~K}, \sigma_{x y}=0$ in a a 2 -Volt interval around $V_{g}^{\prime}=0$.

samples K5, K7, K8 and K29, all measured at $14 \mathrm{~T}$ at $T=0.3 \mathrm{~K}$. For each sample, we have plotted $R_{x x}$ vs. the unshifted gate voltage $V_{g}$, so its peak automatically locates $V_{0}$. It is clear that $\mathrm{K} 7\left(V_{0}=1 \mathrm{~V}\right)$ has the highest peak, followed by $\mathrm{K} 5\left(V_{0}=3 \mathrm{~V}\right)$, whereas $\mathrm{K} 8\left(V_{0}=12\right.$ $\mathrm{V})$ and $\mathrm{K} 29(22.5 \mathrm{~V})$ have peaks that are severely suppressed. Further insight into this pattern of suppression is given below when we examine the $H$ dependence of $R_{0}$ at low $T$.

Sample self-heating may also obscure the divergence. We find that, below $1 \mathrm{~K}$, self-heating becomes serious when the dissipation exceeds $\sim 2 \mathrm{pW}$. The measurements of $R_{x x}$ vs. $V_{g}$ were repeated at 3 currents $(I=0.6,2$ and $15 \mathrm{nA}$ ) at $T=0.3 \mathrm{~K}$. The results at $I=0.6$ and $2 \mathrm{nA}$ are virtually identical. However, the curve at 15 $\mathrm{nA}$ is $30 \%$ smaller near $V_{g}^{\prime}=0$, consistent with heating. Hence, we have kept $I$ at $2 \mathrm{nA}$ to eliminate self-heating as a problem. Heating at the contacts is negligible because of the small contact resistances $(\sim 1 \mathrm{k} \Omega)$ relative to $R_{0}$.

Hereafter, we focus on $R_{0}$, or equivalently, the Dirac-
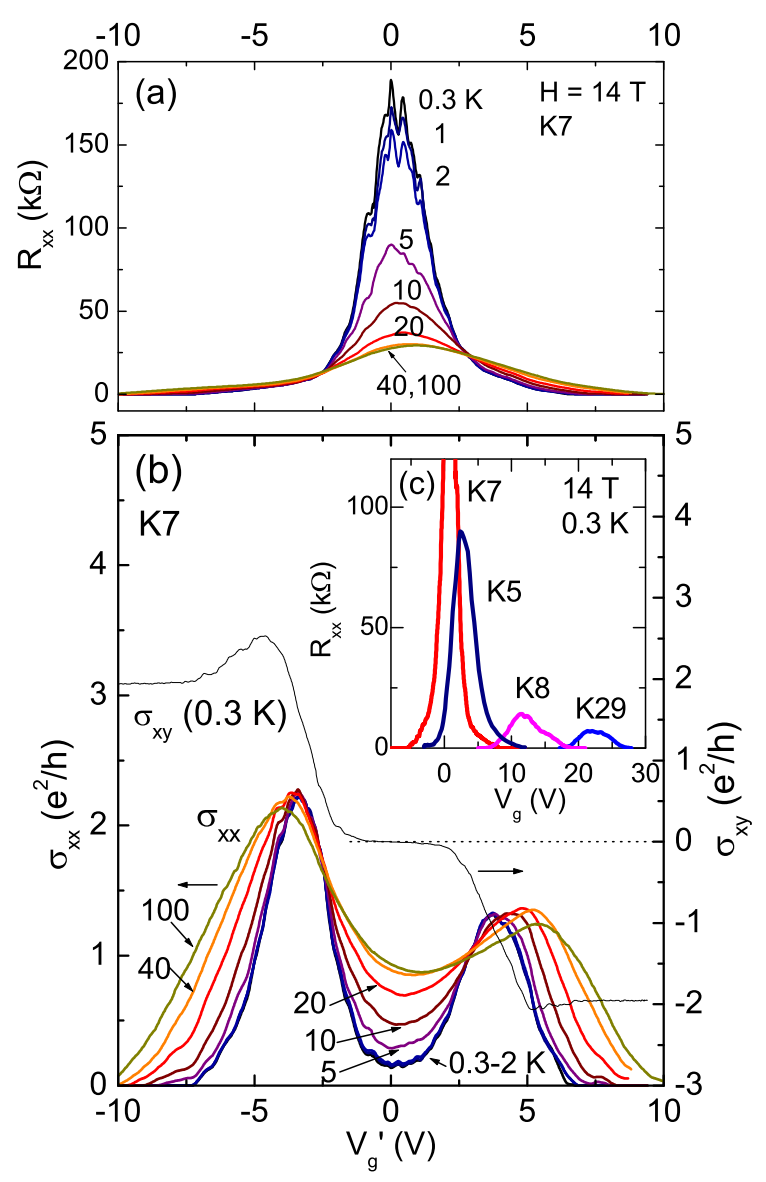

FIG. 2: (color online) The resistance $R_{x x}$, conductivity $\sigma_{x x}$ and the Hall conductivity $\sigma_{x y}$ in $\mathrm{K} 7$ vs. the shifted gate voltage $V_{g}^{\prime}$, with $H$ fixed at $14 \mathrm{~T}$. As $T$ decreases to $0.3 \mathrm{~K}$, the zero-energy peak in $R_{x x}$ (Panel a) rises steeply to $190 \mathrm{k} \Omega$. Panel (b) shows that, as $T$ decreases, double peaks in $\sigma_{x x}$ are clearly resolved. Between the peaks, $\sigma_{x x}$ falls rapidly but saturates below $2 \mathrm{~K}$. The Hall conductivity at $0.3 \mathrm{~K}$ (thin curve) displays a clear plateau $\left(\left|\sigma_{x y}\right|<0.02 e^{2} / h\right)$ in the interval $-1 V<V_{g}^{\prime}<1 V$. Panel (c) compares $R_{x x}$ (of $n=0$ LL) vs. unshifted gate $V_{g}$ in the samples K5, K7, K8 and K29 at $0.3 \mathrm{~K}$. In each sample, $R_{x x}$ peaks at $V_{0}$. As $V_{0}$ increases, $R_{0}(14)$ rapidly decreases.

point conductivity $\sigma_{x x}^{0} \equiv L / w R_{0}(L$ and $w$ are the length and width). Curves of the conductivity versus $\log _{10} T$ are shown in Fig. [3a at selected fields. In low fields $(H<$ $9 \mathrm{~T}$ ), the $T$ dependence of $\sigma_{x x}^{0}$ is quite mild. As $H$ is increased to $14 \mathrm{~T}$, the opening of the gap $\Delta$ (between the $n=0$ sublevels) causes the conductance to decrease sharply below $40 \mathrm{~K}$. However, instead of falling to $0, \sigma_{x x}^{0}$ saturates below $2 \mathrm{~K}$ to a $T$-independent residual value $\sigma_{r e s}$, as anticipated in the discussion of Fig. 2 $\mathrm{b}$. The existence of this residual $\sigma_{r e s}$, which is highly sensitive to $H$, is one of our key findings.

The field dependence of $\sigma_{\text {res }}$ is best viewed as a divergent $R_{0}$. Figure $3 \mathrm{~b}$ shows the rising profile of $R_{0}$ vs. $H$ 

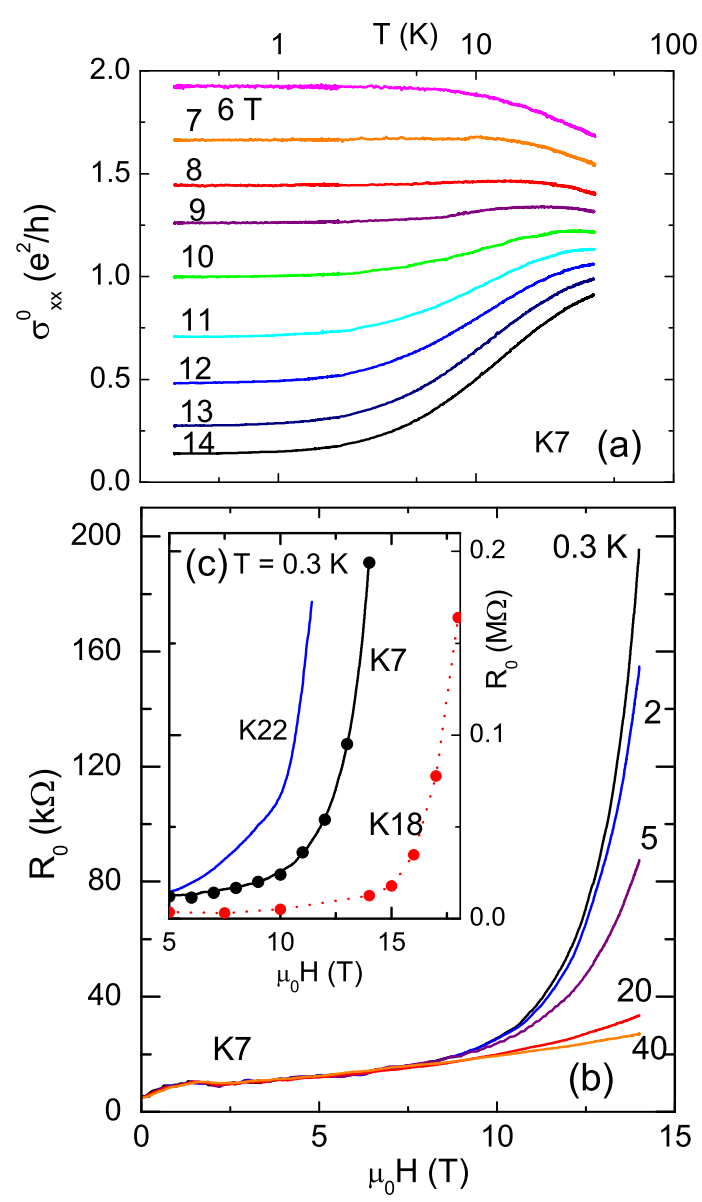

FIG. 3: (color online) The $T$ dependence of $\sigma_{x x}^{0}$ in $\mathrm{K} 7$ (= $\left.L / w R_{0}\right)$ and the $H$ dependence of $R_{0}$ at low temperature. Panel a shows curves of $\sigma_{x x}^{0}$ vs. $\log _{10} T$ with $H$ fixed at 6-14 T. For $H>8 \mathrm{~T}$, the gap $\Delta$ causes $\sigma_{x x}^{0}$ to decrease markedly until saturation at the residual value $\sigma_{\text {res }}$ occurs below $2 \mathrm{~K}$. Panel (b) displays the steep increase in $R_{0}$ vs. $H$ in $\mathrm{K} 7$ at selected $T$. At $0.3 \mathrm{~K}, R_{0}$ appears to diverge at a field near $18 \mathrm{~T}$ (see Fig. 4b). Panel (c) compares the $R_{0}(H)$ profiles in Samples K7, K18 and K22. In Sample K18 ( $\left.V_{0}=20 \mathrm{~V}\right)$, the divergence in $R_{0}$ becomes apparent only above $14 \mathrm{~T}$, whereas in $\mathrm{K} 22\left(V_{0}=-0.6 \mathrm{~V}\right) R_{0}$ starts to diverge at fields lower than in K7. In K7, we have plotted $R_{0}$ values measured by sweeping $V_{g}$ at fixed $H$ (solid symbols) with $R_{0}$ measured by sweeping $H$ with $V_{g}^{\prime}$ fixed at 0 (solid curve), to show consistency.

in sample $\mathrm{K} 7$ at selected temperatures. The divergent form at the lowest $T(0.3 \mathrm{~K})$ strongly suggests that the system is rapidly approaching a field-induced crossover (or transition) to an insulating state.

In light of the importance of $V_{0}$, it is instructive to see how the profile of $R_{0}$ vs. $H$ varies between samples. Figure 3 c compares the results in K7, K18 and K22 at $T=$ $0.3 \mathrm{~K}$. In $\mathrm{K} 18$, where $V_{0}(20 \mathrm{~V})$ is quite large, the divergence in $R_{0}(H)$ becomes noticeable only in fields above $14 \mathrm{~T}$. Conversely, in $\mathrm{K} 22$ for which $V_{0}(-0.6 \mathrm{~V})$ is slightly
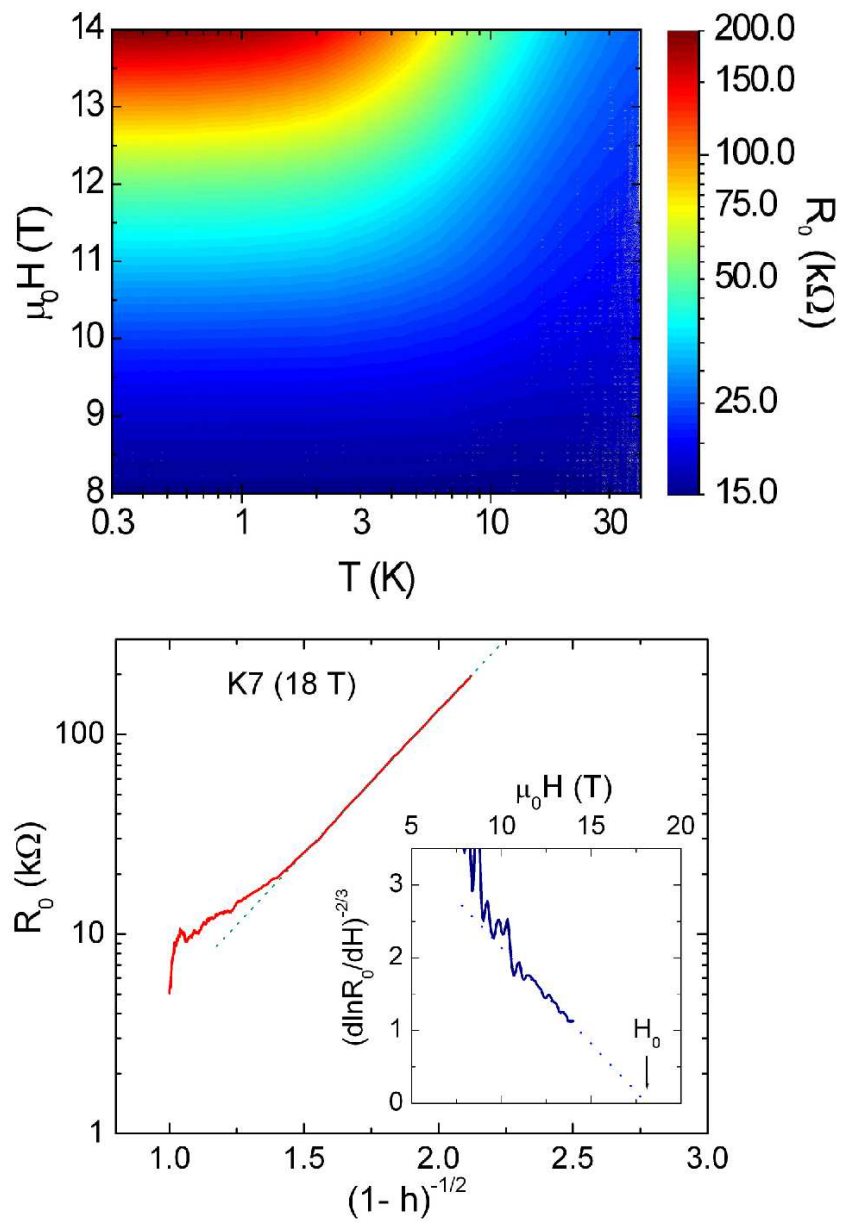

FIG. 4: (color online) (Panel a) The contour plot of $R_{0}(T, H)$ (K7) in the $T-H$ plane (vertical bar shows values of $R_{0}$ ). The contour lines emphasize the unusual approach to the insulating state. At low $T, R_{0}$ is unchanged on a horizontal path $(H$ held fixed), but it rises rapidly on a vertical path (increasing $H$ at fixed $T$ ). Panel (b) displays $\log R_{0}$ vs. $1 / \sqrt{1-h}$ in $\mathrm{K} 7$ at $T=0.3 \mathrm{~K}$, with $h=H / H_{0}$, where $H_{0}=18 \mathrm{~T}$. The linear segment at large $R_{0}$ shows that the divergence is consistent with $R_{0}(h) \sim \exp [2 b / \sqrt{1-h}]$ with $b \sim 0.7$. In Panel (c), the plot of $\left(d \ln R_{0} / d H\right)^{-2 / 3}$ vs. $H$ shows a high-field linear segment that extrapolates to zero at $\sim H_{0}(18 \mathrm{~T})$.

smaller than in $\mathrm{K} 7, R_{0}$ diverges at field scales smaller than in K7. From the trend, it is clear that the divergence in $R_{0}$ is shifted to ever higher fields as $V_{0}$ increases. Referring back to Fig. 2r, we now see that the strong suppression of $R_{0}(14)$ in samples with large $V_{0}$ simply reflects the shift of the divergence to larger $H$. These results underscore the importance of choosing samples with $\left|V_{0}\right|<1 \mathrm{~V}$ for investigating the intrinsic properties of the Dirac-point. In the case of K7, we have also checked that the 2 procedures for measuring $R_{0}$ (varying $V_{g}$ at fixed $H$ or vice versa) give consistent values.

The variation of $R_{0}(T, H)$ in $\mathrm{K} 7$ is conveniently represented in a contour plot in the $T-H$ plane (Fig. 4). 
Below $\sim 2 \mathrm{~K}$, the contour lines are horizontal, which implies that $R_{0}$ is unchanged if the sample is cooled in fixed $H$. This provides evidence that $\sigma_{\text {res }}$ involves gapless excitations. However, if $T$ is fixed, $R_{0}$ rises steeply with $H$, implying proximity to the insulating state (deep-red region). When a system approaches the insulating state, its resistivity generally diverges as $T \rightarrow 0$, as a result of either strong localization (variable-range hopping) or the opening of a mobility gap (weak localization is not relevant here because of the intense $H$ ). In both cases, decreasing $T$ reduces the conductance because the itinerant states are severely depopulated. Hence, the pattern in Fig. 4a is most unusual. The gaplessness of $\sigma_{\text {res }}$ suggests that, below $2 \mathrm{~K}$, these excitations are protected from the effects of changing $T$. Paradoxically, they are not protected from an increasing $H$, which reduces the current carried at an exponential rate.

In the theory in Refs. [15, 18], the current at the Dirac point is carried by a pair of edge states. The change in $R_{x x}$ vs. $H$ is interpreted as an increased scattering rate as the edge states are pushed closer to the edge.

In samples with small $V_{0}$, however, the steep increase in $R_{0}$ to $\sim 200 \mathrm{k} \Omega$ (Fig. 3b) suggests a different regime in which Coulomb exchange may be the dominant energy scale. From Figs. 3b and $4 \mathrm{~b}$, we infer that $R_{0}$ appears to be diverging towards an insulating state in high fields. These considerations lead us to quantify the divergence in $R_{0}$.

We find that $R_{0}$ fits very well to the form $R_{0} \sim$ $\xi(h)^{2}$, where the correlation length $\xi$ has the KosterlitzThouless (KT) form

$$
\xi_{K T} \sim \exp (b / \sqrt{1-h}), \quad\left(h=H / H_{0}\right),
$$

with $H$ replacing $T$. Plotting $\ln R_{0}$ vs. $\sqrt{1-h}$, we find that the high-field portion becomes linear (Fig. 4b) when $H_{0}$ is adjusted to be $17-18 \mathrm{~T}$. From the slope, we find that the parameter $b$ has the value $\sim 0.7$, in agreement with simulations of the KT transition. For self consistency, we may also let the data determine $H_{0}$ by linear extrapola- tion. By Eq. 2, we have $d \ln R_{0} / d H \sim\left(H_{0}-H\right)^{-3 / 2}$. Hence a plot of $\left(d \ln R_{0} / d H\right)^{-2 / 3}$ vs. $H$ should cross the $H$-axis at $H_{0}$. Indeed, this quantity, plotted in Fig. 45, becomes linear at large $H$ and extrapolates to zero at $\sim 18 \mathrm{~T}$ in agreement with (b).

Although consistency with Eq. 2 alone does not prove that a KT transition occurs at $H_{0}$, the fit does reveal the striking exponential character of the divergence in $R_{0}$. We adopt as a working hypothesis that this reflects the approach to a KT transition. The ordered state at large $H$ is destroyed by the spontaneous appearance of defects which increase exponentially in density at $H_{0}$. The steep fall of $R_{0}$ below $H_{0}$ may reflect the current carried by these defects.

In both the QHF state [7, 8, 9, 10, 11] and the excitongap state [13, 14], the 4-fold degeneracy in the $n=0 \mathrm{LL}$ is completely lifted in large $H$ to produce an insulator. In the QHF, the starting symmetry is $S U(4)$ if we treat the spin and pseudospin degrees on equal footing [9]. The reduction of the $S U(4)$ to lower symmetries by the effects of Zeeman energy, disorder or lattice discretization is discussed in Refs. 8, 10, 11, 12]. According to Ref. 11] random disorder may force the pseudospins into the plane. If the ordered state indeed has $U(1)$ symmetry, the XY order is susceptible to a $\mathrm{KT}$ transition [7, 11]. It would be very interesting to relate the fit of $R_{0}$ to Eq. 2 with charged, topological excitations envisioned in the KT transition. Measurements are in progress at higher $H$ to provide further evidence for a transition to the insulating state and to clarify its nature.

We acknowledge valuable discussions with Kun Yang, P. A. Lee, M. Lee, A. Geim and A. Pasupathy. We are indebted to Philip Kim for generously providing Kish graphite crystals. This research is supported by NSFMRSEC under Grant DMR 0213706, and by the Princeton Center for Complex Materials. Some data were taken in the National High Magnetic Field Lab., Tallahassee, which is supported by NSF and the State of Florida.
[1] K. S. Novoselov et al. Science 306, 666-669 (2004).

[2] K. S. Novoselov et al. Proc. Natl. Acad. Sci. USA 102, 10451-10453 (2005).

[3] K. S. Novoselov et al. Nature 438, 197-200 (2005).

[4] Y. Zhang, J. Tan, H. L. Stormer and P. Kim, Nature 438, 201-204 (2005).

[5] Y. Zhang et al. Phys. Rev. Lett. 96, 136806 (2006).

[6] Y.-W. Tan et al. cond-mat/0707.1807 (2007).

[7] K. Nomura and A. H. MacDonald, Phys. Rev. Lett. 96, 256602 (2006).

[8] J. Alicea and M. P. A. Fisher, Phys. Rev. B 74, 075422 (2006).

[9] Kun Yang, S. Das Sarma and A. H. MacDonald, Phys. Rev. B 74, 075423 (2006).

[10] M. O. Goerbig, R. Moessner and B. Douçot, Phys. Rev. B 74, 161407(R) (2006).
[11] D. A. Abanin, P. A. Lee and L. S. Levitov, Phys. Rev. Lett. 98, 156801 (2007).

[12] For a review, see Kun Yang, Solid State Commun. 143, 27 (2007).

[13] D. V. Khveshchenko, Phys. Rev. Lett. 87, 206401 (2001).

[14] V. P. Gusynin, V. A. Miransky, S. G. Sharapov, and I. A. Shovkovy, Phys. Rev. B 74, 195429 (2006).

[15] D. A. Abanin et al. Phys. Rev. Lett. 98, 196806 (2007).

[16] Z. Jiang, Y. Zhang, H. L. Stormer and P. Kim, Phys. Rev. Lett. 99, 106802 (2007).

[17] Sungjae Cho and Michael S. Fuhrer, cond-mat arXiv:0705.3239 2

[18] D. A. Abanin, P. A. Lee and L. S. Levitov, Phys. Rev. Lett. 96, 176803 (2006). 